\title{
Gravitational waves from the 3D collapse of a neutron star to a Kerr black hole
}

\author{
Ian Hawke $^{\mathrm{a}, *}$, Luca Baiotti ${ }^{\mathrm{b}}$, Luciano Rezzolla ${ }^{\mathrm{b}}$, Erik Schnetter ${ }^{\mathrm{a}}$ \\ a Max-Planck-Institut für Gravitationsphysik, Albert-Einstein-Institut, 14476 Golm, Germany \\ b SISSA, International School for Advanced Studies and INFN, Via Beirut 2, 34014 Trieste, Italy
}

Available online 9 April 2005

\begin{abstract}
We present the gravitational waveforms emitted when a neutron star, modeled as an initially uniformly rotating polytrope, collapses to a Kerr black hole. We investigate the gravitational collapse by carefully studying not only the dynamics of the matter, but also that of the trapped surfaces, of both the apparent and event horizons formed during the collapse. The use of these surfaces, together with the dynamical horizon framework, allows for a precise measurement of the black hole mass and spin, as well as of the energy lost to gravitational waves. The ability to extract the waveforms depends on having computational domains with boundaries in the wave-zone and on extending the simulation past the formation of the singularity. Both requirements are met by using fixed mesh-refinement techniques and by excising a region of the computational domain which includes the singularity and is within the apparent horizon.
\end{abstract}

(C) 2005 Elsevier B.V. All rights reserved.

PACS: 04.25.Dm; 04.40.Dg; 04.70.Bw; 95.30.Lz; 97.60.Jd

Keywords: Numerical relativity; Hydrodynamics; Black holes; Neutron stars

The study of the gravitational collapse of rotating stellar configurations leading to black hole formation is a cornerstone of any theory of gravity and a long standing problem in general relativity. Over the years, numerous approaches have been applied and several different techniques developed to tackle the problem. Many of the issues around gravitational collapse are not purely academic. Indeed, fundamental aspects of Einstein's theory, such as the cosmic

\footnotetext{
* Corresponding author.

E-mail address: hawke@aei.mpg.de (I. Hawke).
}

censorship hypothesis or the black hole no-hair theorems, revolve around a complete understanding of gravitational collapse. Furthermore, important issues in relativistic astrophysics awaiting clarification, such as the mechanism responsible for gamma-ray bursts, may be unveiled with a more detailed understanding of the physics of gravitational collapse in rotating and magnetized stars. Last but not least, the investigation of gravitational collapse to rotating black holes can provide the waveforms and the energetics of one of the most important sources of gravitational radiation. 
Although it is evident that it is through numerical relativity simulations that one can hope to improve our knowledge of gravitational collapse under realistic conditions, this is not an easy task. The modeling of black hole spacetimes with collapsing matter-sources in multidimensions is one of the most formidable efforts of numerical relativity. This is due both to the inherent difficulties and complexities of the system of equations which is to be solved (i.e. the Einstein equations coupled to the relativistic hydrodynamics equations) and to the vast computational resources needed in $3 \mathrm{D}$ evolutions.

In addition to the technical difficulties due to the accurate treatment of the hydrodynamics involved in the collapse, the precise calculation of the gravitational radiation emitted in the process is particularly challenging as the energy released in gravitational waves is much smaller than the total rest-mass energy of the system. As an indication of the difficulties inherent in the problem of calculating the gravitationalwave emission in rotating gravitational collapse, only one work dating back to almost 20 years ago [1] is available in the literature on this specific topic. In 1985 Stark and Piran used an axisymmetric generalrelativistic code to evolve rotating configurations and to compute the gravitational radiation produced by their collapse to black holes. The results referred to initial configurations consisting of polytropic stars which underwent collapse after the pressure was reduced by a factor ranging from $60 \%$ up to $99 \%$ for the rapidly rotating models. The initial data effectively consisted of spherically-symmetric solutions with a uniform rotation simply "added" on.

Overall, their investigation revealed that while the nature of the collapse depended on the parameter $a$, the form of the waves remained the same over the entire range of the values of $a$, with the amplitude increasing with $a$. Particularly important was the evidence that the gravitational-wave emission could essentially be related to the oscillations of a perturbed black hole spacetime.

In recent years many studies have extended to three spatial dimensions the investigation of gravitational collapse to black holes [2-4]. Despite the improvements in the evolution of the hydrodynamics and of the spacetime achieved by these simulations, none of them has addressed the problem of the gravitationalwave emission. The reason for this is to be found in the small amplitude of the signal, often below the truncation error of the 3D simulations, but most importantly in the fact that all of the above calculations made use of Cartesian grids with uniform spacing. With the computational resources currently available, this choice places the outer boundaries too close to the source to detect gravitational radiation.

Using a recently developed code for the solution of the Einstein equations in non-vacuum spacetimes, the Whisky code, we have investigated the collapse of rapidly rotating relativistic stars to Kerr black holes [5]. We were able to bound the amount of energy emitted in gravitational waves by detailed study of both the apparent and event horizons. However, as in previous works, these simulations made use of numerical grids with uniform spacing and thus with outer boundaries very close to the initial position of the stellar surface. Under these conditions, the gravitational radiation extracted does not provide interesting information besides the obvious change in the quadrupole moment of the background spacetime.

To allow us to extend our domain into the wave zone and extract gravitational waves we have used progressive mesh-refinement (PMR) techniques. We have adopted a Berger-Oliger prescription for the refinement of meshes on different levels [6] and used the numerical infrastructure described in [7]. In addition to this, we have also implemented a simplified form of adaptivity in which new refined levels are added at predefined positions during the evolution. As the collapse proceeds and the star occupies smaller portions of the computational domain, three more refined levels are added one by one, nested in the four original ones. By the time the simulation is terminated at $\sim 190 \mathrm{M}$, the finest typical spatial resolution is $\Delta x \sim 0.02 M$ whilst the domain extends out to $\sim 82.5 \mathrm{M}$. A detailed discussion of the grid and of its evolution will be given in [8].

The initial data for our simulations is the same described in [5] and basically consists of axisymmetric rotating relativistic stars, calculated as equilibrium solutions of the Einstein equations in a compactified domain and in polar coordinates. For a direct comparison with the results in [1] and because no shock is observed during the collapse, the stars are modeled with a polytropic equation of state (EOS) $p=K \rho^{\Gamma}$, with $\Gamma=2$ and with the polytropic constant which is initially $K_{\mathrm{ID}}=100$. Once secularly unstable solu- 
tions are found along sequences of fixed angular momentum or fixed rest-mass we consider initial models that have the same axes ratios but slightly larger central energy densities and are dynamically unstable (i.e. models D1-D4 in [5]). Hereafter we will restrict the discussion to the collapse of the most rapidly rotating dynamically unstable model, namely D4. Discussions on the collapse of the other models and how the collapse is triggered can be found in $[5,8]$.

While several different methods are possible for the extraction of the gravitational-radiation content in numerical spacetimes, we have adopted a gaugeinvariant approach in which the spacetime is matched with the non-spherical perturbations of a Schwarzschild black hole. In practice, a set of "observers" is placed on 2-spheres of fixed coordinate radius $r_{\mathrm{ex}}$, where they extract the gauge-invariant, even $\Psi_{\ell m}^{(\mathrm{e})}$ and odd-parity $Q_{\ell m}^{(\mathrm{o})}$ metric perturbations [9]. Here $\ell, m$ are the indices of the angular decomposition and we usually compute modes up to $\ell=5$ with $m=0$.

Although the position of such observers is arbitrary and the information they record must be the same for waves extracted in the wave-zone, we place our observers between $40 \mathrm{M}$ and $50 \mathrm{M}$ from the centre of the grid, as done in [1]. The reasons for this are simple: firstly, very little of the gravitational waves has reached the outer boundary by the time the simulation is terminated; secondly, the wave amplitude is progressively reduced as it propagates outward, thus making the extraction increasingly more difficult.

Using the even and odd-parity perturbations $Q_{\ell m}^{+}$ and $Q_{\ell m}^{\times}$(see [9]) we show in Figs. 1 and 2 the lowestorder multipoles for $Q_{\ell m}^{+}$with the offset produced by the stellar quadrupole removed [8]. Fig. 1 refers to the $\ell=2$ mode as extracted by four different observers at increasing distances and expressed in retarded time. Fig. 2 instead refers to the $\ell=4$ mode, with the inset giving a comparison between the two modes and showing that the gravitational-wave signal is essentially quadrupolar, with the $\ell=2$ mode being about an order of magnitude larger than the $\ell=4$.

The very good overlap of the waveforms measured at different positions is important evidence that the extraction has been performed in the wave-zone, since the invariance under a retarded-time scaling is a property of the solutions of a wave equation. A similar

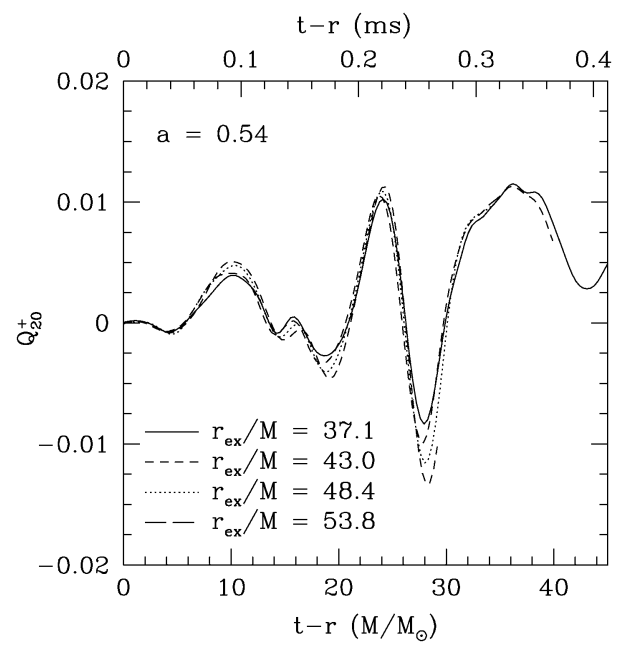

Fig. 1 . The $\ell=2$, even-parity perturbation as extracted by observers at different positions $r_{\mathrm{ex}}$ expressed in retarded time. The overlap of the waves is an indication that the extraction is correct.

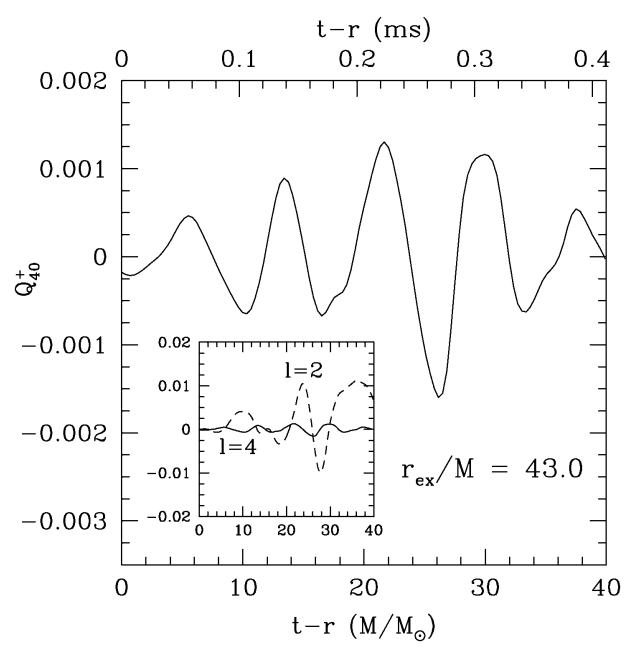

Fig. 2 . The $\ell=4$, even-parity perturbation can also be extracted but has much lower amplitude than the $\ell=2$, as shown in the inset.

overlap is seen also for the $\ell=4$ mode and disappears when the waves are extracted at smaller radii.

Another indication that the waveforms in Fig. 1 are an accurate description of the gravitational radiation produced by the collapse comes by analyzing their power spectra. The collapse, in fact, can be viewed as the rapid transition between the spacetime of the initial equilibrium star and the spacetime of the produced rotating black hole. It is natural to expect, therefore, that the waveforms produced in this process will reflect the 


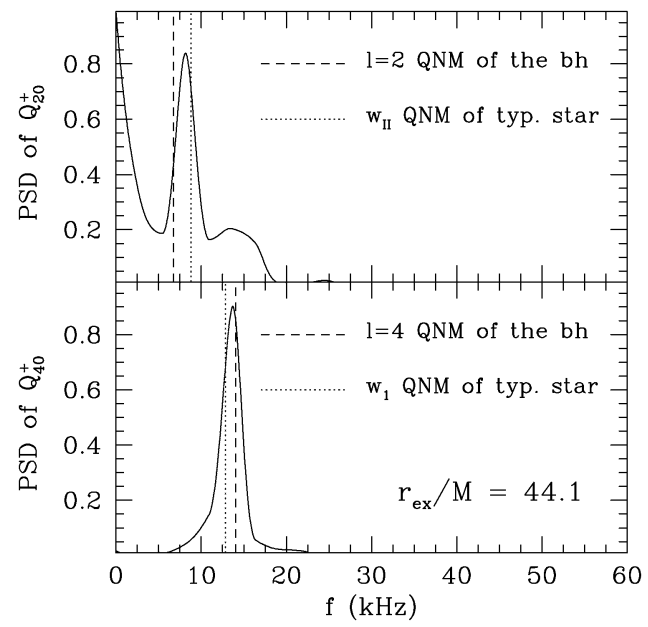

Fig. 3. Power spectra of the waveforms reported in Fig. 1. The dashed vertical lines indicate the frequencies of the QNMs of a black hole, while the dotted ones the $w_{\mathrm{I}}$ and $w_{\mathrm{II}}$ modes of a typical star.

basic properties of both spacetimes and in particular the fundamental frequencies of oscillation. We validate this in Fig. 3, where we show the power spectral densities (PSD) of the waveforms of the metric perturbations $Q_{20}^{+}$and $Q_{40}^{+}$reported in Figs. 1 and 2 (the units on the $y$-axis are arbitrary). The upper panel of Fig. 3 shows the PSD of $Q_{20}^{+}$and compares it with the frequencies of the $\ell=2, m=0$ quasi-normal mode (QNM) of a Kerr black hole with $M=1.861 M_{\odot}$ and $a=0.6$ (dashed line at $6.7 \mathrm{kHz}$ ) [10] as well as with the first $w_{\text {II }}$ "interface" mode for a typical compact star with $M=1.27 M_{\odot}$ and $R=8.86 \mathrm{~km}$ (dotted line at $8.8 \mathrm{kHz}$ ) [11]. Similarly, the lower panel of Fig. 3, shows the PSD of $Q_{40}^{+}$comparing it with the $\ell=4$, $m=0$ QNM of a Schwarzschild black hole (dashed line at $14.0 \mathrm{kHz})[11]$ and the first $w_{\mathrm{I}}$ "curvature" mode (dotted line at $12.8 \mathrm{kHz}$ ) [11]. The excellent agreement between the position of these peaks and the fundamental frequencies of the vacuum and nonvacuum spacetimes is an important confirmation of the robustness of the results obtained.

In conclusion, we have shown that the accurate extraction of gravitational radiation is now possible also in numerical relativity simulations making use of 3D grids with Cartesian coordinates. The great potential shown by the PMR techniques employed here opens the way to a number of applications that would be otherwise intractable with uniform grids. Work is now in progress to consider initial models with realistic EOSs or in differential rotation, for which values $a \gtrsim 1 \mathrm{can}$ be reached and a more intense gravitational radiation is expected.

\section{Acknowledgements}

It is a pleasure to thank V. Ferrari, K. Kokkotas, N. Stergioulas, O. Zanotti for useful discussions, and C. Ott and B. Zink for help with the PMR algorithm. The computations were performed on the clusters $\mathrm{Al}$ bert 100 at the University of Parma and Peyote at the Albert Einstein Institute. ES acknowledges funding by the DFG's SFB TR/7.

\section{References}

[1] R.F. Stark, T. Piran, Gravitational-wave emission from rotating gravitational collapse, Phys. Rev. Lett. 55 (1985) 891.

[2] M. Shibata, T.W. Baumgarte, S.L. Shapiro, Stability and collapse of rapidly rotating, supramassive neutron stars: 3D simulations in general relativity, Phys. Rev. D 61 (2000) 044012.

[3] M. Shibata, Axisymmetric general relativistic hydrodynamics: Long-term evolution of neutron stars and stellar collapse to neutron stars and black holes, Phys. Rev. D 67 (2003) 024033.

[4] M.D. Duez, S.L. Shapiro, H.-J. Yo, Relativistic hydrodynamics evolutions with black hole excision, Phys. Rev. D 69 (2004) 104016

[5] L. Baiotti, I. Hawke, P.J. Montero, F. Löffler, L. Rezzolla, N. Stergioulas, J.A. Font, E. Seidel, Three-dimensional relativistic simulations of rotating neutron star collapse to a Kerr black hole, Phys. Rev. D 71 (2005) 024035, gr-qc (0403029).

[6] M.J. Berger, J. Oliger, Adaptive mesh refinement for hyperbolic partial differential equations, J. Comp. Phys. 53 (1984) 484-512.

[7] E. Schnetter, S.H. Hawley, I. Hawke, Evolutions in 3D numerical relativity using fixed mesh refinement, Class. Quantum Grav. 21 (6) (2004) 1465-1488.

[8] L. Baiotti, I. Hawke, L. Rezzolla, E. Schnetter, Gravitationalwave emission from rotating gravitational collapse in three dimensions (2005), in preparation.

[9] V. Moncrief, Gravitational perturbations of spherically symmetric systems. I. The exterior problem, Ann. Phys. 88 (1974) 323-342.

[10] E. Leaver, An analytic representation for the quasi-normal modes of Kerr black holes, Proc. R. Soc. London A 402 (1985) 285-298.

[11] K.D. Kokkotas, B.G. Schmidt, Quasi-normal modes of stars and black holes, Living Rev. Relativ. 2 (1999) 1999-2000. 